\title{
The Choice of Jurisprudence and Human Relationship-On the Tragic Implication and Aesthetic Value of the Kunqu Opera Bai Luo Shan*
}

\author{
WU Xiu-Ming \\ Nanjing Normal University,Nanjing, China
}

\begin{abstract}
Bai Luo Shan (白罗衫) is called “Hamlet” in Chinese opera. Bai Luo Shan was originally a legend written by anonymous people in the Ming Dynasty. It is based on the novel Warning the World·Su Zhi Xian Luo Shan Zai He (警世通言·苏知县罗衫再合), Which tells the story of Sun Yun, which occupies a place in the history of opera and performance. Since the Tang Dynasty, the story of Bai Luo Shan has been widely known among the people, and it has been adpated into a script by a variety of operas. Among them, the Kunqu Opera version of Bai Luo Shan dealing with the protagonist's dilemma in the face of jurispudence and human relationship shows the contradiction of human nature, strengthens the tragic meaning of the theme, and embodies the unique aesthetic value.
\end{abstract}

Keywords: Bai Luo Shan, tragic consciousness, aesthetic value

Bai Luo Shan is a well-known folk story from the Tang Dynasty to the Qing Dynasty. It firstly appeared in the legend of Tang Dynasty: Wen Qi Lu·Li Wenmin (闻奇录·李文敏), Qian Zhuan Zi· Chen Yilang (乾馔子·陈 义郎), Yuan Hua Ji·Cui Weizi (原化记·崔尉子). The legend of Bai Luo Shan, written by anonymous in the Ming Dynasty, is the origin of many dramas. Jin Yue Kao Zheng (今乐考证)、Chuan Qi Pin (传奇品)、Qu Kao (曲 考)、Qu Hai Mu (曲海目)、Qu Lu (曲录) includes it which is named Luo Shan Ji (罗衫记). It’s written a story that Xu Neng, a water bandit, muedered Su Yun and wanted to forcibly occupy his wife and adopt his posthumous. Many years later, Su Yun's posthumous knew the truth and revenged finally.

\section{The Evolution of the Story of Bai Luo Shan}

The matrix of Chinese Classical Opera says “the matrix of opera refers to the smallest'plot unit' or a single 'narrative unit' in the opera works, which has a stable inheritance and common attributes and characteristics, sometimes it is also a type of image, symbolic metaphor or prototype" (Wang, 2015, p. 1). The study of the opera matrix is helpful to analyze the reflection of social and cultural background in opera works, the evolution law of opera text and the character change of characters.

\footnotetext{
* Acknowledgements: this paper is funded by National Social Science Fund major bidding project“Compilation and literature research of the Qing dynasty opera” (11\&ZD107).

WU Xiu-ming, Ph.D. candidate, School of Classical Literture, Nanjing Normal University, Nanjing, China.
} 
The story of Bai Luo Shan is a common type of story in traditional opera and novel. Since the Tang Dynasty to the end of the Qing Dynasty, it has experienced the evolution from folklore to script. The script derived from this story is still the representative work in Peking Opera, Kunqu Opera and other plays. The story of Bai Luo Shan is first seen in three legends of the Tang Dynasty included in Tai Ping Guang Ji (太平广记). The story of Li Wenmin, the story of Chen Yilang and the story of Cui Weizi contained in Wen Qi Lu, Gan Zhuan Zi and Yuan Hua Ji. These three legendary novels are the source of their theme.

One is the story of Li Wenmin in Wen Qi Lu. Li Wenmin, born in the Tang Dynasty, went to Guangzhou for a government official position of surpervising called Lu Shi Can Jun. On the way, he was murdered by the bandits and thrown in the river. The robbers replaced the official position of Guangzhou and forcibly occupied his wife and five-year-old son. When Li Wenmin's son grew up and went to capital for an examination, he met an old woman on the East Road of Weinan county. The old woman saw him wearing the white shirt, recognized him and told him about his father's surffering. So he quitted the exam and went back home to confirm his father's matter. His mother told him all and he reported it to the government. Finally his stepfather was punished. The story is relatively simple. The main characters are Li Wenmin, Li Wenmin's son, the thief and Li Wenmin's mother. This story has the slight difference with Bai Luo Shan, but they both take white shirt as the introduction and publicize the theme of karma.

The second is Chen Yilang's story in Gan Zhuan Zi. During the Tianbao period of Tang Dynasty, Chen Yishuang, a native of Fuchang, Dongluo, and Zhou Maofang studied together. Yishuang got the high score during the imperial examination and married Guo Xin as his wife. He went to his post at Pengzhou, Zhou Maofang, his fellow villager, accompanied him. Zhou Maofang was jelous about him and intended to murder him about 500 hundred miles away from Yilong. Zhou let his servant go ahead and walked with Chen Yishuang. Suddenly, When they went through the rugged and narrow path, Zhou struck Yishuang's head with a golden mallet, then pretended that Chen died because his frightened horse stepped into the river and cheated Chen's families to replace Chen's official pisition, and induced Zhou's families to agree that he would replace Chen's official postition and cheated Chen's wife Guo and two-year-old son of Yi Shuang. Seventeen years later, Yilang went for the imperial examination. On the way, he met an old woman who had compassion for her son like her grandson and sent him with the Blood-stained shirt left by Yilang's mother. After Yilang went home, his mother saw the Blood-stained shirt and told him the truth, Yilang was indignant, waited for Zhou fall into sleep, he assassinated him with a dagger. The government official sentenced him unguilty and he carried his mother back to the east at last. Chen Yilang's story is relatively complete, similar to the plot of the Bai Luo Shan, but the person who went to the post was killed by his fellow countryman, not a water thief.

The third is the story of Cui Weizi in Yuan Hua Ji. During the Tianbao period of the Tang Dynasty, Cui's family, who lived in Qinghe, Xingyang, Henan Province, went to Jizhou to work and took Sun Yun's boat. On the way, Sun pushed Cui down into the deep pool and raped Cui's wife Wang. Later Wang give birth to a boy. The boatman looted this baby as his own son and lives in Jiangxia. Twenty years later, when the son went to the imperial examination, lost his way at night, he went to a village and met a granny. This old woman cried bitterly after seeing his apperance which extremly looked like her son. When he came back, he visited the old woman again and received food and clothes donated by her. When he reached home, his mother saw the clothes and told him the truth. He sued Sun to the court and Sun was punished finally. 
Although the contents of the above three stories are slightly different, the core plot is all about the victims on the way to the office - the robbers occupy the wife and son — the official's son and the old mother recognize each other through clothes - the official's son revenge. Moreover, the three legendary robbers all love their adopted son. This kind of story matrix has already possessed the basic plot of the story of Bai Luo Shan, and also provided the plot backbone for the later storytelling novels and legends.

In the Yuan Dynasty, the story of Zhang Guobin's drama Han Shan Ji (汗衫记) changed the legend of Tang Dynasty in the theme and character set. This drama tells Chen Hu was taken in by Zhang Xiaoyou's family. Later, Chen Hu took a fancy to Li Yu'e, the wife of Zhang Xiaoyou. He pretended to persuade Zhang Xiaoyou to take his wife to divine the long pregnancy. On the way, Chen Hu pushed Zhang Xiaoyou into the river and took Zhang's wife. Eighteen years later, Zhang's son won the first place in the martial arts examination. After his mother told him what happened, he found Zhang Xiaoyou through with his shirt, captured Chen Hu and reunited with his family. The secular life of the Yuan Dynasty was found in the story of Han Shan Ji, such as Chen Hu was driven out of debt and Zhang Xiaoyou loaned money, the plot was more rational, Chen Hu didn't love the son, and his posthumous son was hostile to his stepfather, at the end of the play, Zhang Xiaoyou was not dead, and his son was revenged and reunited with his family, which was in line with the narrative mode of happy ending of traditional Chinese opera.

At the end of Ming Dynasty, Feng Menglong's story Warning the World·Su Zhi Xian Luo Shan Zai He, stated that in the Yongle period of the Ming Dynasty, Sun Yun, the eldest son of Sun family in Zhuozhou was appointed as Lanxi County Magistrate of Jinhua Prefecture, Zhejiang Province. On the way to Lanxi, he took a robber Xu Neng's boat due to his shabby and leaky boat and was robbed by that gangster and thrown into the water. His wife was looted but later released by Xu Yong, the robber Xu Neng’s younger brother. Su's wife gave birth to Sun's baby on the way to escape but had to abandon him and hide herself in a nunnery. Xu Neng found the baby during chasing and took him back home to adopt and name him as Xu Jizu. Su Yun was saved and henceforth taught in a village. When Xu Jizu grew up to be fifteen and went for the imperial examination, he met his grandma and saw the white shirt. Later Xu was appointed as Supervisory Censor, and Mrs. Su filed her complain to Xu. Xu questioned Su's originial housekeeper Yao Da and learned the truth. Finally the thief was punished. Xu Jizu changed his name to Su Tai and the whole family was reunited.

In contrast, the plot of the novel is more tortuous and the relationship between the characters is more complex. The frame of the story is basically fixed. First, the characters are basically fixed: Su Yun, Xu Neng, XuJizu, Yao Da. Second, the environmental place names are basically fixed: Zhuozhou, Lanxi and other places.Third, the plot conflicts are more concentrated: the script focuses on the encounter of the Su Yun family. In addition to the continuation of the plot of "being murdered", "meeting" and "Reunion", the increasing of the plot of "rescue", "pitching”, “confession" and "trial in the court” make the story more ups and downs, and the contradictions and conflicts are greatly strengthened.

The related themes of Ming and Qing legends include Yuan Shan Tang Qu Pin (远山堂曲品) which describes zhou Jilu’s He Shan Ji (合衫记), Qu Pin (曲品) which records Shen jing’s He Shan Ji, etc. By the end of the Qing Dynasty, the related origami in Kun Qu Da Quan (昆曲大全), Ji Cheng Qu Pu (集成曲谱), Zhui Bai Qiu (缀白装) and other books were still performed on the stage. In the Ming and Qing Dynasties, there were also drum lyrics of Bai Luo Shan, the plot of which was basically the same with that of the novel. 
Up to now, the script adapted from the story of Bai Luo Shan is still the representative repertoire of Peking opera, Kunqu Opera, Bang Zi opera and so on. To a certain extent, Bai Luo Shan of Kunqu Opera version goes beyond the traditional mode, and strengthens the tragic meaning of the theme by mining the choice between “jurisprudence” and "human feelings” of the protagonist.

Besides the theme of the story, Bai Luo Shan also includes many factors such as politics, law, religion, ethics and morality. For example, when new officials go to their posts, imperial edicts are robbed and previous officials lose their posts. There are often complex social reasons for robbers' presence, such as years of war, famine, large-scale annexation of land, displacement of people, etc. In ancient law, the standard of dealing with robbers was very strict. For example, according to the "robber" article in the 19th volume of the Tang Dynasty code, "all the powerful robbers take their wealth with their power"and "those who steal according to the law, even if they are irrelevant people, have to be caught to send a lawsuit" (Zhangsun, 1983, p. 356). According to the "robber" article of the 18th "criminal law one" in the Ming Dynasty law, "all the robbers who have already done something but can't make money will have one hundred sticks and expel three thousand kilometers. whoever gains wealth, will be beheaded" (Huai, 1999, p. 140). The article of "day plunder" says: "Whoever plunders people's belongings in the day shall have a staff of one hundred for three years...Intentional wounding, behead. Not a mastermind should be reduced by one grade and stabbed the word "snatch" on his right arm" (Huai, 1999, p. 140).

In ancient novels and operas, a large number of robber's works of murdering man and forcibly his wife emerged. On one hand, it reflected the seriousness of robber's problem in ancient social life, and reflected the reality of social chaos and legal runaway. On the other hand, it also showed that the mode had a far-reaching influence in novels and operas, because of its unique twists and turns of plot, especially quite dramatic. The mutation of the effect (the crime, the mystery of life experience, the reunion of family members, the detective) is easy to attract the interest of readers and audience, so it has always been favored by novelists and dramatists.

\section{The Tragic Meaning and Aesthetic Value of the Kunqu Opera Bai Luo Shan}

Westerners divide drama into tragedy and comedy. According to Aristotle's poetics, "tragedy is an imitation of a serious, complete and long action.Its medium is language, with all kinds of melodious sounds, which are used in each part of the play respectively,the way of imitation is to express this emotion by the action of the characters, rather than by the narrative method, by arousing pity and fear" (Aristotle, 1997, p. 19). According to Aristotle, tragedy is a complete organic whole, the root of the tragedy is the plot, second is the character.

Wang Guowei’s History of Opera in Song and Yuan Dynasty hold that “after the Ming Dynasty, legends were nothing but comedies, and there were tragedies in Yuan Dynasty. The most tragic ones are Guan Hanqing's The Injustice of DouE Yuan (窦娥冤) and Ji Junxiang's The Orphan of Zhao (赵氏孤儿).... Even in the world's tragedies, they are not inferior" (Wang, 1995, p. 120). From the perspective of the opera theme, Wang Guowei thinks that DouE Yuan and The Orphan of Zhao can be comparable to Western tragedies. Zeng Yongyi's The Formation and Classification of Chinese Classical Opera (中国古典戏曲的形成和类别)said,” Western drama is based on the philosophy of life, expressing the internal and external aspects of life. Chinese drama originated from the people, with the purpose of ethical enlightenment and entertainment... most of them are tragicomedies” (Zeng, 1975, p. 10). 
Kunqu Opera Bai Luo Shan sets up a mainline of Xu Jizu, which connects the whole story with his passive discovery of the mystery of his life experience. The contradiction focuses on Xu Jizu's choice of kinship and benevolence, legal principle and personal feelings. Xu Jizu in Kunqu Opera can neither understood nor solved the problem when he was faced with the dilemma between the kindness of upbringing and the importance of blood. Finally, the suicide of his adoptive father solved the dilemma for Xu Jizu.

In the novel Warning the World, Xu Jizu knows that Xu Neng is not only a loving father, but also a bandit who has done bad things, but he is dedicated to ethicality. When he learns that Xu Neng murdered his biological father, Xu Jizu arrested Xu Neng and his accomplices without hesitation. After that, he asked biological parents to watch the punishment and changed their names to return to their ancestors. The image of Xu Jizu in the novel is more powerful than that in the opera, but he thinks completely in the prescribed framework, and lacks the three-dimensional sense of the character image.

Xu Neng's image in the novel is quite different from that in Kunqu Opera. In the novel, he can attach importance to the brotherhood. Even if Xu Yong, his younger brother, let Mrs. Su escape, he would not be investigated. But he never stopped murdering and robbery. In the opera, Xu Neng not only gave up his vicious behaviour but also does good deeds. He only wants to stay with Xu Jizu and enjoy his family's happiness. It is because of the change of Xu Neng's image that Xu Jizu has a great impact on his heart when facing his relatives and enemies. What he suffered was a fight between two emotions.

The tragedy is a kind of aesthetic experience in essence. It aroused the resonance of the receiver and the experience of the sublime between the reader and the audience. The ultimate expression of tragedy is the insurmountable contradiction and conflict, that is the destructive force. The "destructive power" in Bai Luo Shan comes from Xu Neng's last chance to escape, but he can only lament powerlessly that "it's better to be a robber than to ask you to read and be an official” (Chen, 2013, p. 90), which is the regret of a robber and the sorrow of a father.

In social ethics, kinship is not only the feeling of blood, but also the feeling of kindness. How to deal with the adoptive father who has nurtured his kindness for 18 years? Compassion makes him unable to bear the punishment according to law.The heart of Jurisprudence forces him to punish crimes according to law and revenge his father's murdered. It is the contradiction that can not be solved constitutes the tragedy character of $\mathrm{Xu}$ Jizu, even if the ending is justice and family reunion.

Bai Luo Shan adoptes the basic framework of Tang legend and script for story-telling, and has made great achievements in plot arrangement, character-shaping, language expression, etc. Kunqu Opera Bai Luoshan strengthens the focus of the drama on the choice between "legal principle" and "human emotion", and increases the tragic meaning. Its character relationship and theme are in line with human care and modern ethical values, which is a modern interpretation of a new value concept.

\section{References}

Aristotle. (1997). Poetics. Beijing: People’s education press.

Chen, Y. (2013). One hunred pieces of Kunqu Opera. Shanghai: Shanghai Classical Publishing House.

Huai, X. F. (1999). Law of the Ming Dynasty . Beijing: Law Press.

Wang, G. W. (1995). The history of the song and Yuan dynasty Opera. Shanghai:East China Normal University Press.

Wang, Z. (2015). The matrix of Chinese classical opera. Beijing: China Social Sciences Press. 
Zeng, Y. Y. (1975). Chinese classical drama anthology. Taibei: Lianjing Publishing Company.

ZhangSun, W. J. (1983). Tang Lv Shu Yi. Beijing: Zhonghua Book Company. 